\title{
The Comparative Molecular Epidemiology of GII.P7_GII.6 and GII.P7_GII.7 Norovirus Outbreaks in Victoria, Australia, 2012-2014
}

\author{
Leesa D. Bruggink Jean M. Moselen John A. Marshall \\ Victorian Infectious Diseases Reference Laboratory, Melbourne, Vic., Australia
}

\section{Key Words}

Gll.6 norovirus · Gll.7 norovirus · Gastroenteritis outbreaks ·

Age $\cdot$ Australia

\begin{abstract}
The comparative molecular epidemiology of the related GII.P7_GII.6 and GII.P7_GII.7 noroviruses has not been examined in detail. ORF 1, ORF 2 and ORF 1/ORF 2 RT-PCR as well as sequencing and phylogeny analysis were carried out on faecal specimens from 873 gastroenteritis outbreaks in Victoria, Australia (2012-2014). There were 575 (66\%) detected as positive for norovirus by means of ORF 1 RT-PCR and/or ORF 2 RT-PCR. Of these, 24 (4.2\%) were Gll.6 (ORF 2) outbreaks, 7 (1.2\%) were Gll.7 (ORF 2) outbreaks, and 1 outbreak $(0.2 \%)$ involved both GII.6 (ORF 2) and GII.7 (ORF 2) noroviruses. The median age of patients identified with GII.6 (ORF 2) (84 years) was significantly different from that of patients identified with GIl.7 (ORF 2) (39 years). ORF 2 Gll.6 and ORF 2 Gll.7 sequences were always associated with a GII.P7 ORF 1 sequence, and GII.P7 sequences fell into two clusters, with one corresponding to the Gll.6 ORF 2 genotype and the other to the GII.7 ORF 2 genotype, thereby indicating that the ORF 1 has been evolving separately for the two viruses. Thus, two closely related noroviruses can have a markedly different incidence and epidemiology.

(c) 2016 S. Karger AG, Basel
\end{abstract}

The noroviruses, which are now considered one of the major causes of gastroenteritis worldwide [1], are singlestranded positive-sense RNA viruses classified as the genus Norovirus within the family Caliciviridae [2]. Noroviruses have been classified into 6 genogroups, and 3 of these, genogroups I, II and IV (GI, GII and GIV), occur in human infections [2]. The noroviruses can be further subdivided into genotypes [2]; the GII.4 genotype appears to be the most common in humans [3].

The human norovirus genome comprises 3 open reading frames (ORFs) [2]. ORF 1 encodes the non-structural polyprotein, ORF 2 encodes the major capsid protein and ORF 3 encodes a minor capsid protein [2]. Norovirus genotype classification using nucleic acid sequencing can be based on the ORF 1 region or the ORF 2 region [4]. Recombination often occurs in the region of the junction of ORF 1 and ORF 2, so that recombination can result in differing genotype classifications for ORF 1 and ORF 2 in a given genome [5].

Although GII.6 (ORF 2) and GII.7 (ORF 2) noroviruses have been documented in various studies [6-8] and GII.6 (ORF 2) has been noted to be a relatively common norovirus in some settings [9-12], detailed molecular epidemiological analysis of these viruses is lacking. Furthermore, although a link between GII.6 and GII.7 noroviruses has been documented, in that both can be linked to

\section{KARGER}

E-Mail karger@karger.com

www.karger.com/int
(C) 2016 S. Karger AG, Basel

0300-5526/16/0591-0060\$39.50/0
Dr J.A. Marshall

Victorian Infectious Diseases Reference Laboratory

Doherty Institute, 792 Elizabeth Street

Melbourne, VIC 3000 (Australia)

E-Mail john.marshall@ mh.org.au 
GII.P7 [13-16], the precise relationship between GII.P7_ GII.6 and GII.P7_GII.7 does not appear to have been examined. This study reviews the molecular epidemiology of GII.P7_GII.6 and GII.P7_GII.7 noroviruses in gastroenteritis outbreaks in Victoria, Australia, and examines the relationship between the two.

For the purposes of the study, an outbreak of gastroenteritis was defined as an incident, apparently associated with a common event or location, in which $\geq 4$ individuals had symptoms of gastroenteritis [17].

All outbreaks included in the study were those for which faecal specimens were sent to the Victorian Infectious Diseases Reference Laboratory (VIDRL) for the testing of outbreaks that occurred during the period 2012-2014. VIDRL is the main public health laboratory for viral identification in the state of Victoria, Australia. As such, it receives faecal material from gastroenteritis outbreaks reported to the Victorian Department of Health. Outbreak specimens are also occasionally sent by other institutions such as hospitals. Outbreak documentation and management were carried out by staff linked to the Victorian Department of Health and/or staff at the affected setting. Only outbreaks that occurred in Victoria were included in the study.

The date of an outbreak was taken as the onset date. If this was not available, the date the outbreak was first notified or the earliest date of collection of a specimen from the outbreak was taken as the date of the outbreak. Only one faecal specimen per person per outbreak was tested.

For the purposes of analysis, norovirus outbreak settings were classified as either 'healthcare' or 'non-healthcare'. Healthcare outbreaks included the following settings: age care facility, disabled care facility and hospital. Non-healthcare outbreaks included the following settings: school camp, child-care centre, children's activity centre, gathering, navy base, consumption of suspect food, consumption of suspect water and restaurant.

For the period 2012-2014, 3307 faecal specimens from 873 gastroenteritis outbreaks were tested for noroviruses. Faecal specimens were prepared as a $20 \%$ (vol/vol) suspension in Hanks' complete balanced salt solution, and clarified by centrifugation [18]. RNA extraction was then carried out using the Corbett (now Qiagen GmbH, Hilden, Germany) automated nucleic acid extraction procedure [19].

Four norovirus RT-PCR protocols were utilised in the study. Protocol 1 was an ORF 1 RT-PCR which involved a 2-round, hemi-nested procedure, using the primers NV4562, NV4611, NV4692, NV5296, NV5298 and NV5366. It was carried out essentially as given previous-

GII.P7_GII.6 and GII.P7_GII.7 Norovirus Outbreaks ly [20, 21]. Protocol 2 involved the use of a GII ORF 2 region C RT-PCR procedure. It utilised primers G2F3 and G2SKR and was carried out essentially as given previously $[22,23]$. Protocol 3 involved the use of a GII ORF 1 RT-PCR procedure. It utilised primers MON 431 and MON 433 and was carried out essentially as given previously [24, 25]. Protocol 4, a GII ORF 1/ORF 2 RT-PCR that crosses the junction of ORF 1 and ORF 2, was developed for this study and was carried out to confirm the presence of recombinant forms. The RT-PCR utilised the primers MON 431 and G2SKR in a two-round procedure. The first round comprised an RT step carried out at $50^{\circ} \mathrm{C}$ for $30 \mathrm{~min}$, followed by PCR with initial denaturation at $94^{\circ} \mathrm{C}$ for $5 \mathrm{~min}$, then 30 cycles of denaturation at $94^{\circ} \mathrm{C}$ for $30 \mathrm{~s}$, annealing at $50^{\circ} \mathrm{C}$ for $1 \mathrm{~min}$ and extension at $72^{\circ} \mathrm{C}$ for $3 \mathrm{~min}$. An additional extension step was then carried out at $72^{\circ} \mathrm{C}$ for $10 \mathrm{~min}$. The second-round PCR was performed as for the first round but without the RT step.

Nucleotide sequencing was carried out for the products of the RT-PCR protocols 2, 3 and 4 essentially as given previously [26]. For protocol 2, a fragment of 195 bp corresponding to nucleotides 5,133-5,327 relative to the norovirus reference strain JX459908 was used. For protocol 3, a fragment of $105 \mathrm{bp}$ corresponding to nucleotides 4,880-4,984 relative to the norovirus reference strain JX459908 was used. For protocol 4, a fragment of 368 bp corresponding to nucleotides $4,937-5,304$ relative to the norovirus reference strain JX459908 was used.

Phylogenetic analysis of the nucleotide sequences was carried out using MacVector software v8.0 (Oxford Molecular Limited, Madison, Wis., USA). Genotype identification was carried out using the norovirus typing tool of Kroneman et al. [27]. Where the typing tool was unable to type the fragment, a BLAST search was performed and the closest match was put into the typing tool to identify the genotype. Phylogenetic trees were constructed using Phylip v3.68, essentially as described previously [26], to examine the clustering patterns of ORF 1 and ORF 2 fragments.

During the period 2012-2014, all faecal specimens from gastroenteritis outbreaks received at VIDRL were tested by RT-PCR protocol 1. In addition, 1 specimen chosen at random from each RT-PCR protocol 1 negative outbreak was then tested by the ORF 2 RT-PCR (protocol 2). Where a GII.6 or GII.7 ORF 2 genotype was detected, all specimens in that outbreak were tested by the protocol 2 RT-PCR and all positive specimens were then sequenced and phylogenetic analysis was carried out. As sequencing analysis indicated that RT-PCR protocol 1 did not normally detect GII.P6 or GII.P7, all specimens positive for 


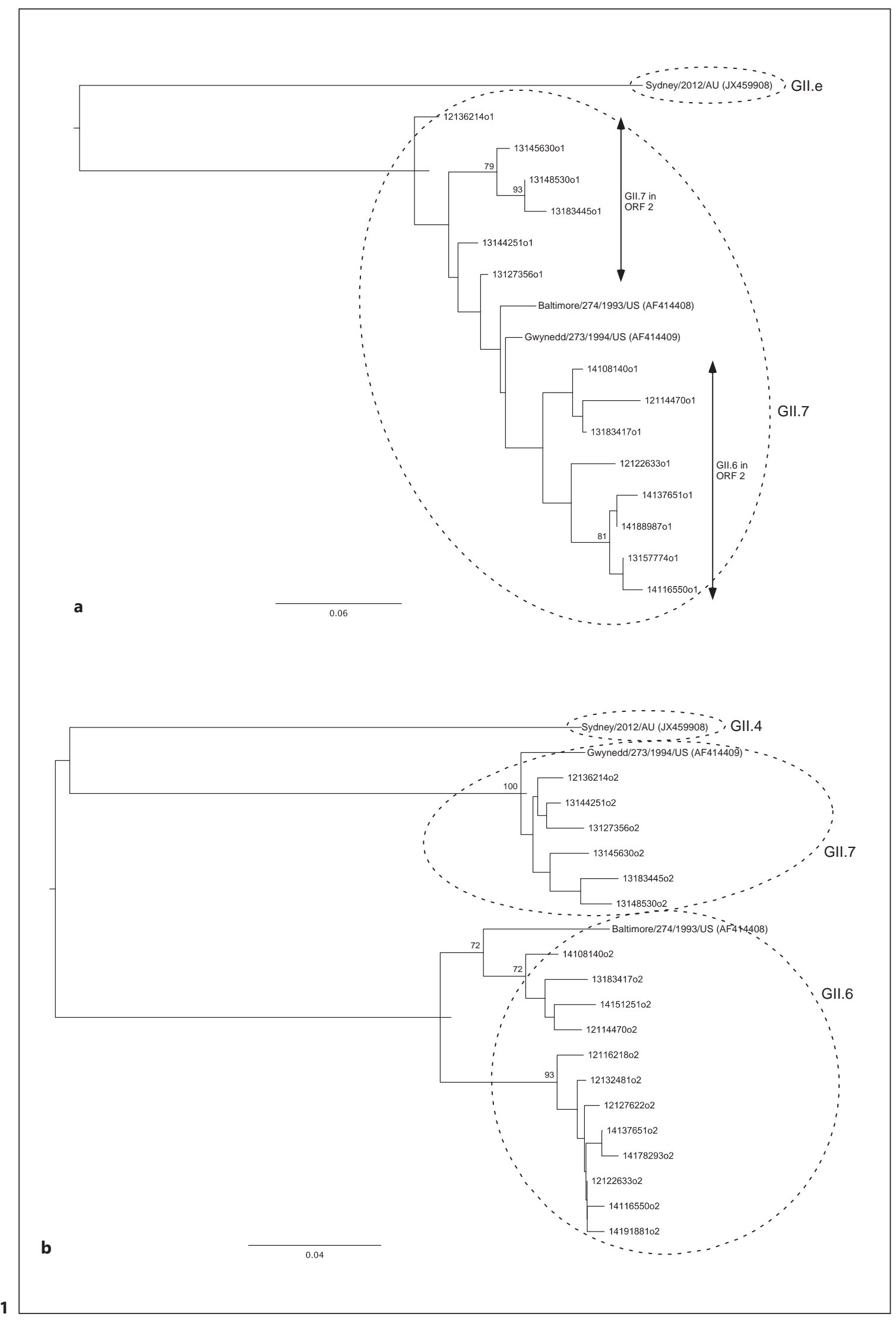

(For legend see next page.) 
ORF 2 GII.6 or GII.7 were tested by ORF 1 RT-PCR protocol 3, an alternate ORF 1 procedure, which can detect ORF 1 GII.P7. All specimens positive for ORF 1 GII.P7 norovirus were then subjected to sequencing and phylogenetic analysis. In order to confirm the presence of the ORF 1/ORF 2 forms, GII.P7_GII.6 and GII.P7_GII.7, 1 specimen for each of these forms was tested by the ORF 1/ORF 2 RT-PCR protocol 4 and sequenced.

Faecal specimens were received from 873 gastroenteritis outbreaks and 575 (66\%) were positive for norovirus by either the protocol 1 ORF 1 RT-PCR or the protocol 2 ORF 2 RT-PCR. Of these, 24 (4.2\%) were GII.6 (ORF 2) outbreaks, 7 (1.2\%) were GII.7 (ORF 2) outbreaks and 1 outbreak (0.2\%) involved both the GII.6 (ORF 2) and GII.7 (ORF 2) noroviruses. Thus GII.6 norovirus outbreaks were about 3 times more common than GII.7 norovirus outbreaks. This difference was statistically significant $\left(\mathrm{p}<0.005, \chi^{2}\right.$ test; the calculation excluded the outbreak with both GII.6 and GII.7). Both GII.6 and GII.7 could occur in a variety of healthcare and non-healthcare settings and there was no statistical difference in the incidence of GII.6 and GII.7 outbreaks in healthcare compared to non-healthcare settings.

Application of the Kolmogorov-Smirnov method [28] indicates that the age distribution of all patients with GII.7 (ORF 2) was normal ( $\mathrm{p}>0.20$ ) whereas the age distribution of all patients with GII.6 (ORF 2) was not normal ( $\mathrm{p}<0.01)$. The median age for the GII.6 patients was 84 years $(n=56$; range $1-98$ years; interquartile range 54.75 years) and the median age for GII.7 patients was 39 years $(n=65$; range $1-89$ years; interquartile range 37.0 years). Application of the median test [28] showed that the median age of those with GII.6 was significantly different to that of those with GII.7 ( $p=0.027)$. Thus there

Fig. 1 a DNA (dist) Kimura phylogenetic tree showing the phylogenetic relationship of all unique GII.P7 ORF 1 sequences detected. The tree was based on a 105-bp ORF 1 sequence corresponding to nucleotides $4,880-4,984$ relative to the norovirus reference strain JX459908. The reference strains included in the tree were: Baltimore/274/1993/US (AF414408) (GII.P7_GII.6), Gwynedd/373/1994/US (AF414409) (GII.P7_GII.7) and Sydney/2012/ AU (JX459908) (GII.Pe_GII.4). b DNA (dist) Kimura phylogenetic tree showing the phylogenetic relationship of all unique GII.6 and GII.7 ORF 2 sequences detected. The tree was based on a 195bp ORF 2 sequence corresponding to nucleotides 5,133-5,327 relative to the norovirus reference JX459908. The reference strains included in the tree were as for $\mathbf{a} . \mathbf{a}, \mathbf{b}$ The figures on the branches represent bootstrap values (\%) after resampling 1,000 data sets. Only bootstrap values $\geq 70 \%$ are shown. The scale marker represents substitutions per site.

GII.P7_GII.6 and GII.P7_GII.7 Norovirus Outbreaks was a difference in the epidemiology of GII.6 (ORF 2) and GII.7 (ORF 2) outbreaks.

ORF 1 RT-PCR and sequencing were successfully carried out by protocol 3 in 28 of the 32 outbreaks; in the 4 outbreaks where an ORF 1 sequence could not be obtained, 3 had a GII.6 ORF 2 genotype and 1 had a GII.7 ORF 2 genotype. Application of the norovirus typing tool, in combination with BLAST analysis, indicated that all ORF 1 sequences linked to the ORF 2 GII.6 and GII.7 genotypes belonged to the ORF 1 genotype GII.P7. The phylogenetic tree corresponding to all unique ORF 1 sequences found in association with ORF 2 GII.6 and GII.7 noroviruses is shown in figure 1a. The phylogenetic tree corresponding to all unique ORF 2 GII.6 and GII.7 sequences is shown in figure $1 \mathrm{~b}$. A comparison shows that the upper cluster in the ORF 1 phylogenetic tree (fig. 1a) corresponded to GII.7 in ORF 2 (fig. 1b) and the lower cluster (fig. 1a) corresponded to GII.6 in ORF 2 (fig. 1b). These observations indicate that GII.P7_GII.6 and GII. P7_GII.7 have been evolving separately for some time.

The protocol 4 ORF 1/ORF 2 bridging RT-PCR procedure, in combination with sequence analysis, confirmed the identity of the GII.P7_GII.7 form and of the recombinant GII.P7_GII.6 form; 2 such representative sequences have been lodged in GenBank as KT970472 and KT970473, respectively.

This study is one of the first to examine the incidence, molecular epidemiology and relationship between GII.6 and GII.7 noroviruses linked to the GII.P7 ORF 1 genotype. In general, the findings indicate that these 2 viruses are relatively rare in gastroenteritis outbreaks in Victoria, Australia, and that they both occurred in a broad range of settings. However, although both genotypes affected individuals across a wide range of ages, GII.6 norovirus tended to occur more commonly in older people whereas GII.7 norovirus occurred more in younger people. This suggests that the viruses circulate in different ways in the community and, consequently, that their epidemiology may be different.

The results of our study indicate that GII.6 norovirus was more common than GII.7 norovirus, and this is in close agreement with the findings of Zhirakovskaia et al. [29], who examined sporadic gastroenteritis in children in Russia in the period 2003-2012. They found GII.6 in 20 faecal samples tested whereas GII.7 was only found in 3 faecal samples. In contrast, Tan et al. [8], in a study of norovirus in children in Nanning City, China, in 20102011, found that GII.7 (3/84) was only slightly more common than GII.6 (2/84), although the low number of cases here makes it impossible to draw any conclusions about

Intervirology 2016;59:60-65 
the relative frequency of the 2 genotypes from this study. Therefore, the evidence indicates that, in general, GII.6 norovirus is more common than GII.7 norovirus.

Two GII.6 recombinant forms have been described previously. GII.P7_GII.6 has been identified in a number of studies around the world including China [15], South Africa [13] and Uruguay [14]. Truong et al. [30] suggested they had identified the recombinant GII.P4_GII.6 in South Korea, but this finding remains to be confirmed. Our study documents the first identification of the recombinant form GII.P7_GII.6 in Australia and no other ORF 1 recombinant forms linked with ORF 2 GII.6 have been found.

A key observation is that both capsid (ORF 2) genotypes, the GII.6 and GII.7 noroviruses, were linked to the same ORF 1 genotype, GII.P7. An examination of the ORF 1 GII.P7 phylogenetic tree indicated that all GII.P7 sequences in this study fell into two clusters and that one cluster corresponded to the GII.6 ORF 2 genotype and the other cluster to the GII.7 ORF 2 genotype. This suggests that the recombination events occurred some time ago, and that since then the ORF 1 regions have evolved separately for these 2 viruses.

The findings of this study enable some speculation about the evolution of the noroviruses. Since the recombination of GII.P7 with GII.6, the 2 norovirus genotypes, GII.P7_GII.6 and GII.P7_GII.7, appear to have been evolving away from each other. This is possibly facilitated by differences in their epidemiology. It is notable that one of these viruses, GII.P7_GII.6, is the more successful.

\section{References}

1 Arias A, Emmott E, Vashist S, Goodfellow I: Progress towards the prevention and treatment of norovirus infections. Future Microbiol 2013;8:1475-1487.

2 Green KY: Caliciviridae: the noroviruses; in Knipe DM, Howley PM (eds): Fields Virology, ed 6. Philadelphia, Wolters Kluwer/Lippincott Williams \& Wilkins, 2013, vol 1, pp 582-608.

3 de Graaf M, van Beek J, Vennema H, Podkolzin AT, Hewitt J, Bucardo F, Templeton K, Mans J, Nordgren J, Reuter G, Lynch M, Rasmussen LD, Iritani N, Chan MC, Martella V, Ambert-Balay K, Vinje J, White PA, Koopmans MP: Emergence of a novel GII.17 norovirus - end of the GII.4 era? Euro Surveill 2015;20:21178.

4 Kroneman A, Vega E, Vennema H, Vinje J, White PA, Hansman G, Green K, Martella V, Katayama K, Koopmans M: Proposal for a unified norovirus nomenclature and genotyping. Arch Virol 2013;158:2059-2068.

5 Bull RA, Tanaka MM, White PA: Norovirus recombination. J Gen Virol 2007;88:33473359.

6 Alam A, Qureshi SA, Vinje J, Zaidi A: Genetic characterization of norovirus strains in hospitalized children from Pakistan. J Med Virol 2016;88:216-223.

7 Luo LF, Qiao K, Wang XG, Ding KY, Su HL, Li CZ, Yan HJ: Acute gastroenteritis outbreak caused by a GII.6 norovirus. World J Gastroenterol 2015;21:5295-5302.

8 Tan D, Deng L, Wang M, Li X, Ma Y, Liu W: High prevalence and genetic diversity of noroviruses among children with sporadic acute gastroenteritis in Nanning City, China, 20102011. J Med Virol 2015;87:498-503.
9 Andrade JdSR, Rocha MS, Carvalho-Costa FA, Fioretti JM, Xavier MdPTP, Nunes ZMA, Cardoso J, Fialho AM, Leite JPG, Miagostovich MP: Noroviruses associated with outbreaks of acute gastroenteritis in the state of Rio Grande do Sul, Brazil, 2004-2011. J Clin Virol 2014;61:345-352.

10 Chan-It W, Thongprachum A, Khamrin P, Kobayashi M, Okitsu S, Mizuguchi M, Ushijima $\mathrm{H}$ : Emergence of a new norovirus GII.6 variant in Japan, 2008-2009. J Med Virol 2012;84:1089-1096.

11 Paklonskaya NU, Amvrosieva TV, Dziadziulia KL, Baranouskaya NM, Kishkurno EP, Kluiko NL: Norovirus infection in Belarus: occurrence and molecular epidemiology. Cent Eur J Public Health 2015;23:69-72.

12 Sakon N, Yamazaki K, Nakata K, Kanbayashi D, Yoda T, Mantani M, Kase T, Takahashi K, Komano J: Impact of genotype-specific herd immunity on the circulatory dynamism of norovirus: a 10-year longitudinal study of viral acute gastroenteritis. J Infect Dis 2015;211: 879-888.

13 Mans J, Murray TY, Taylor MB: Novel norovirus recombinants detected in South Africa. Virol J 2014;11:168.

14 Fajardo A, Tort FL, Victoria M, Fumian TM, Miagostovich MP, Leite JPG, Cristina J, Colina R: Phylogenetic analyses of norovirus strains detected in Uruguay reveal the circulation of the novel GII.P7/GII.6 recombinant variant. Infect Genet Evol 2014;28:328-332.
15 Wu X, Han J, Chen L, Xu D, Shen Y, Zha Y, Zhu X, Ji L: Prevalence and genetic diversity of noroviruses in adults with acute gastroenteritis in Huzhou, China, 2013-2014. Arch Virol 2015;160:1705-1713.

16 Sakon N, Yamazaki K, Yoda T, Tsukamoto T, Kase T, Taniguchi K, Takahashi K, Otake T: Norovirus storm in Osaka, Japan, last winter (2006/2007). Jpn J Infect Dis 2007;60:409410 .

17 Bruggink L, Sameer R, Marshall J: Molecular and epidemiological characteristics of norovirus associated with community-based sporadic gastroenteritis incidents and norovirus outbreaks in Victoria, Australia, 2002-2007. Intervirology 2010;53:167-172.

18 Witlox KJ, Karapanagiotidis T, Bruggink LD, Marshall JA: The effect of fecal turbidity on norovirus detection by reverse transcriptase polymerase chain reaction. Diag Microbiol Inf Dis 2010;66:230-232.

19 Witlox KJ, Nguyen TN, Bruggink LD, Catton MG, Marshall JA: A comparative evaluation of the sensitivity of two automated and two manual nucleic acid extraction methods for the detection of norovirus by RT-PCR. J Virol Methods 2008;150:70-72.

20 Yuen LKW, Catton MG, Cox BJ, Wright PJ, Marshall JA: Heminested reverse multiplex transcription-PCR for detection and differentiation of Norwalk-like virus genogroups 1 and 2 in fecal samples. J Clin Microbiol 2001; 39:2690-2694.

21 Bruggink LD, Oluwatoyin O, Sameer R, Witlox KJ, Marshall JA: Molecular and epidemiological features of gastroenteritis outbreaks involving genogroup I norovirus in Victoria, Australia, 2002-2010. J Med Virol 2012;84: 1437-1448. 
22 McIver CJ, Bull RA, Tu ETV, Rawlinson WD, White PA: Norovirus and Sapovirus; in McIver CJ (ed): A Compendium of Laboratory Diagnostic Methods for Common and Unusual Enteric Pathogens - An Australian Perspective. Melbourne, Australian Society for Microbiology, 2005, pp 191-198.

23 Bruggink LD, Dunbar NL, Marshall JA: Emergence of GII.e as a major ORF 1 norovirus genotype and its associated ORF 2 GII.4 variant forms. Infect Genet Evol 2014;22: 157-163.

24 Vinje J, Vennema H, Maunula L, von Bonsdorff C-H, Hoehne M, Schreier E, Richards A, Green J, Brown D, Beard SS, Monroe SS, de Bruin E, Svensson L, Koopmans MPG: Inter- national collaborative study to compare reverse transcriptase PCR assays for detection and genotyping of noroviruses. J Clin Microbiol 2003;41:1423-1433.

25 Bruggink LD, Witlox KJ, Sameer R, Catton MG, Marshall JA: Evaluation of the RIDAQUICK immunochromatographic norovirus detection assay using specimens from Australian gastroenteritis incidents. J Virol Methods 2011;173:121-126.

26 Bruggink LD, Marshall JA: Molecular and epidemiological features of GIIb norovirus outbreaks in Victoria, Australia, 2002-2005. J Med Virol 2009;81:1652-1660.

27 Kroneman A, Vennema H, Deforche K, Avoort Hvd, Penaranda S, Oberste MS, Vinje
J, Koopmans M: An automated genotyping tool for enteroviruses and noroviruses. J Clin Virol 2011;51:121-125.

28 Siegel S: Nonparametric Statistics for the Behavioral Sciences. New York, McGraw-Hill Book Company, 1956.

29 Zhirakovskaia EV, Tikunov AY, Bodnev SA, Klemesheva VV, Netesov SV, Tikunova NV: Molecular epidemiology of noroviruses associated with sporadic gastroenteritis in children in Novosibirsk, Russia, 2003-2012. J Med Virol 2015;87:740-753.

30 Truong TC, Than VT, Kim W: Evolutionary phylodynamics of Korean noroviruses reveals a novel GII.2/GII.10 recombination event. PLoS One 2014;9:e113966. 